\title{
Short communication: A countrywide survey of antimicrobial- resistant indicator bacteria in Kosovo's dairy farms
}

\author{
Jeffrey C. Chandler, ${ }^{\star} \dagger$ Sulaiman F. Aljasir,† Afrim Hamidi,‡ Driton Sylejmani,‡ Kenneth G. Gerow,§ \\ and Bledar Bishat ${ }^{1}$ \\ *National Wildlife Research Center, Wildlife Services, Animal and Plant Health Inspection Service, USDA, Fort Collins, CO 80521 \\ †Department of Animal Science, University of Wyoming, Laramie 82071 \\ †Faculty of Agriculture and Veterinary, University of Prishtina, 10000 Prishtina, Kosovo \\ $\S$ Department of Statistics, University of Wyoming, Laramie 82071
}

\begin{abstract}
The World Health Organization recently recognized the Republic of Kosovo as one of the highest consumers per capita of antibiotics for human use among nonEuropean Union Eastern European countries; however, data are limited regarding antimicrobial usage and antimicrobial resistance in the livestock sector for this recently formed country. The objective of this study was to conduct the first nationwide survey of antimicrobial resistance phenotypes in indicator bacteria collected from dairy farms in Kosovo. Composite fecal samples were collected from 52 farms located within all 7 administrative districts of Kosovo in the summer of 2014. Isolation and characterization of the indicator bacteria Escherichia coli $(\mathrm{n}=165)$ and Enterococcus spp. $(\mathrm{n}=153)$ from these samples was achieved by culturing on selective/differential media with and without select antibiotics, followed by MALDI-TOF (matrixassisted laser desorption/ionization time-of-flight) mass spectrometry-based identification and antimicrobial susceptibility testing using the disk diffusion method. When no selective pressure was applied in culture-based isolation, the majority of E. coli and Enterococcus spp. collected were resistant to $\leq 1$ of 16 and $\leq 2$ of 12 antibiotics tested, respectively. In contrast, E. coli and Enterococcus spp. isolated using sub-minimum inhibitory concentrations of cefoxitin, ciprofloxacin, or erythromycin were typically resistant to at least one and often multiple antibiotic types, which primarily consisted of certain $\beta$-lactams, quinolones, sulfonamides, phenicols, and tetracyclines for E. coli isolates and macrolides, tetracyclines, and rifamycins for enterococci isolates.
\end{abstract}

Key words: antimicrobial resistance, Escherichia coli, Enterococcus spp., Kosovo

Received November 3, 2017.

Accepted April 18, 2018.

${ }^{1}$ Corresponding author: bbisha@uwyo.edu

\section{Short Communication}

Antimicrobial resistance (AMR) is expected to be one of the greatest challenges faced by animal agriculture and public health (US CDC, 2013; WHO, 2014). Globally, large quantities of antimicrobials are used for human therapy and agricultural applications, promoting AMR development in the diverse microbial communities associated with these systems (US CDC, 2013; US FDA-NARMS, 2014; US FDA, 2016). Animal production is recognized as the primary agricultural driver of AMR, as antimicrobials are administered therapeutically, prophylactically, and for growth promotion (US FDA-NARMS, 2014; US FDA, 2016). When coupled with estimates of increased antimicrobial use in countries with growing animal production over the next decade, this suggests further expansion of the livestockassociated AMR threat (Van Boeckel et al., 2015).

The AMR problem is largely unaddressed in low- to middle-income countries, exposing critical gaps within AMR monitoring and mitigation networks (Founou et al., 2016). Countries with weak or inadequate national policies, regulation, and surveillance systems were identified by the World Health Organization (WHO) to be at increased risk for producing AMR bacteria as well as for dissemination of these bacteria internationally (WHO, 2014). The Republic of Kosovo is a recently formed country in the Balkan Peninsula of Europe. A lack of regional collaboration, coordination, and resources have limited Kosovo's ability to control the use of antimicrobials and monitor AMR. Estimates compiled by the WHO ranked Kosovo's population to be the fourth highest consumer of antibiotics among 13 non-European Union Eastern European countries surveyed, with the majority of human antibiotic use attributed to $\beta$-lactams (including cephalosporins), quinolones, macrolides, and sulfonamides (Versporten et al., 2014). Knowledge of AMR in Kosovo's livestock sector is more limited, but $\beta$-lactams, sulfonamides, and tetracyclines are reported to be the most widely 
administered antibiotics in Kosovo's cattle (Sulejmani et al., 2012; Gallina et al., 2013), and 3 studies suggest this use may be reflected in the AMR phenotypes of associated bacteria (Mehmeti et al., 2015; Hamidi and Sylejmani, 2016; Mehmeti et al., 2016).

The objective of this study was to conduct the first nationwide survey of priority AMR phenotypes within the indicator bacteria Escherichia coli and Enterococcus spp. collected from dairy farms in Kosovo. These bacteria were specifically chosen for analyses because they are reported to have antimicrobial susceptibility fingerprints that are somewhat reflective of net antibiotic exposure in their environment (Harwood et al., 2000; Lillehaug et al., 2005), resistance determinants found within these genera can be associated with mobile genetic elements (Frye and Jackson, 2013), and these bacteria are used to broadly represent resistance trends for gram-negative and gram-positive organisms in animal production, respectively (US FDA-NARMS, 2014).

A 2014 agricultural census indicated that 134,393 head of dairy cattle are present within 63,874 agricultural holdings in Kosovo's 7 administrative districts (ASK, 2015). The population of dairy cows and agricultural holdings within these districts were reported as Prishtinë (28,834 cows, 14,592 holdings), Mitrovicë (16,740 cows, 8,159 holdings), Pejë (21,856 cows, 8,889 holdings), Prizren (22,862 cows, 12,252 holdings), Ferizaj (11,673 cows, 6,359 holdings), Gjilan (12,623 cows, 5,005 holdings), and Gjakovë (19,805 cows, 8,168 holdings; ASK, 2015). Although not specifically classified in the agricultural census, a 2007 report indicates the average dairy herd size in Kosovo was 1.6 head per agricultural holding, indicating the majority of dairy farms were for subsistence (US AID, 2007). Only 6\% of Kosovo's dairy farms were classified as commercial operations (defined as $\geq 5$ head per holding; US AID, 2007).

In this study, E. coli and Enterococcus spp. were isolated from composite fecal samples, collected from 52 commercial dairy farms intentionally selected to cover all 7 administrative districts of Kosovo in July 2014 (Table 1). Farm accessibility, herd size ( $\geq 5$ animals), and geographic distribution were the primary factors used to direct sampling efforts. The sample was not randomly selected, and so summary values from it cannot be formally used as estimates of countrywide parameters; we provide them as conjectural estimates only. Logistic regression was used to determine the odds ratio of target isolates displaying AMR to the priority antibiotics tested in this study [cefoxitin (FOX); ciprofloxacin (CIP), and erythromycin (ERY)] to 3 epidemiological variables including herd size, breed, and sampling location. A 95\% confidence interval was used to assess correlation of multidrug resistance of isolates with herd size, breed, and sampling location. Herd size was rescaled (size divided by 10) for analysis so that a 1-unit increase corresponds to an additional 10 animals. This is because, for instance, herds of size 47 and 48 are essentially the same size, whereas 47 and 57 are appreciably different. All statistical analyses were performed using Minitab version 16 (Minitab Inc., State College, PA).

The composite fecal samples consisted of 10 fresh individual fecal samples collected from pen floors (10 fecal samples per farm). Samples were stored on ice until laboratory analyses were initiated, which occurred on the day of collection. The individual fecal samples were homogenized via manual mixing to prepare the composite sample. Composite samples were then directly inoculated onto 8 different agar medium formulations, which included Enterococcosel agar (ENT; Becton Dickinson, Sparks, MD) or MacConkey agar (MAC) (Becton Dickinson) with and without supplemented sub-MIC of antibiotics, and incubated overnight at $37^{\circ} \mathrm{C}$. The antibiotics used were CIP (Sigma-Aldrich, Saint Louis, MO), $1 \mu \mathrm{g} / \mathrm{mL}$; FOX (Sigma-Aldrich), 4 $\mu \mathrm{g} / \mathrm{mL}$; and ERY (Sigma-Aldrich), $4 \mu \mathrm{g} / \mathrm{mL}$. These concentrations of antibiotics were specifically chosen to minimize microbial stresses in the transition to agar media, while still providing limited selective pressure. Single isolates were then propagated in brain heart infusion broth (Becton Dickinson) overnight at $37^{\circ} \mathrm{C}$, mixed with $40 \%$ glycerol (Sigma-Aldrich), and stored at $-80^{\circ} \mathrm{C}$ until further analyses could be performed.

Isolates were confirmed as E. coli or Enterococcus spp. by matrix-assisted laser desorption/ionization biotyping following a standard formic acid-acetonitrile extraction procedure (Bizzini et al., 2010) using a Bruker Microflex LRF mass spectrometer pre-calibrated with bacterial test standard operated with Bruker Biotyper software (ver. 3.1) (Bruker, Billerica, MA). Specieslevel identifications were only accepted if scored $\geq 2.0$ by the Biotyper algorithm. To examine the resistance phenotypes of confirmed E. coli and Enterococcus spp. isolates, antimicrobial susceptibility testing using the disk diffusion method was performed in accordance with Clinical and Laboratory Standards Institute guidelines (CLSI, 2015). Specifically, Sensi-Discs (Becton Dickinson) were used to evaluate antimicrobial susceptibilities to 16 and 12 antibiotics for E. coli and Enterococcus spp., respectively. This screening encompassed 11 classes of antibiotics for E. coli and 10 classes of antibiotics for Enterococcus spp.

The 52 dairy farms sampled in this study contained between 5 and 220 head of cattle, primarily of Holstein and Simmental breeds, with an average of 32.5 head per farm (Table 1). Two farms exclusively reared sheep and 


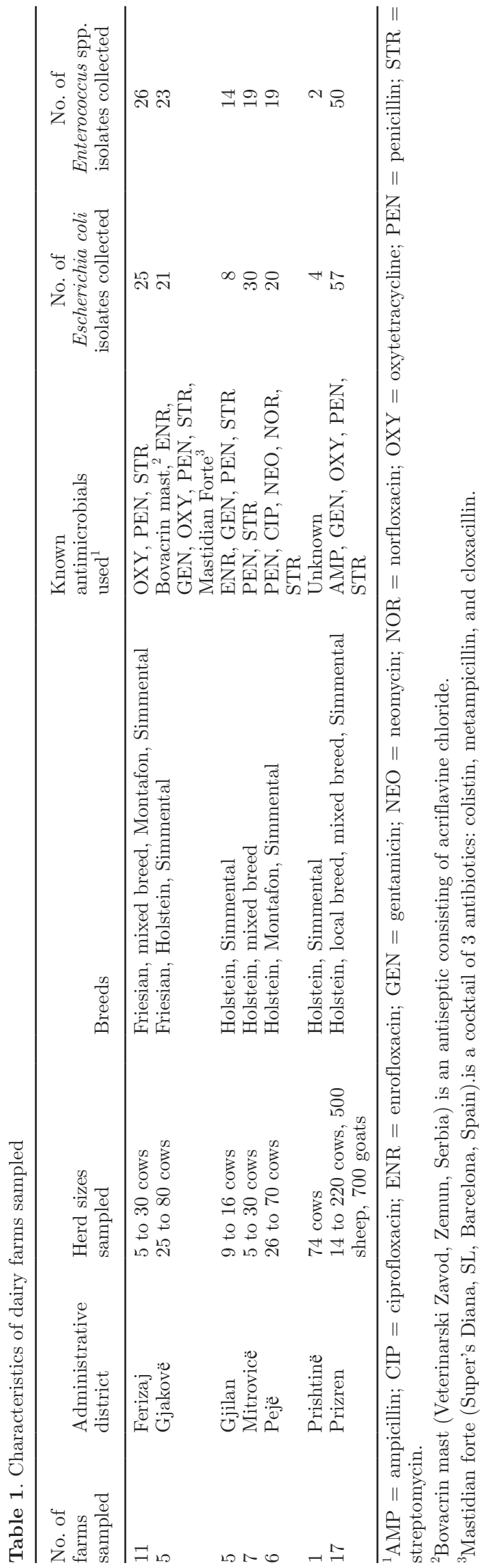

goats. All but 8 farms reported antimicrobial use, with penicillin (29 farms, 56\%) and streptomycin (28 farms, $54 \%$ ) being the most frequently reported antimicrobials used. Use of gentamicin (4 farms, $8 \%$ ), oxytetracycline (10 farms, 19\%), and enrofloxacin (5 farms, 10\%) was reported on multiple farms sampled.

From a total of 447 presumptive isolates, 165 and 153 isolates of E. coli and Enterococcus spp. were obtained, respectively. Non-E. coli and Enterococcus spp. isolates were excluded from additional analyses. Ten different species of enterococci were identified, with Enterococcus faecalis being the most frequently encountered species $(\mathrm{n}=63)$, followed by Enterococcus faecium $(\mathrm{n}=44)$, Enterococcus hirae $(\mathrm{n}=25)$, Enterococcus mundtii $(\mathrm{n}=$ 9), Enterococcus pseudoavium $(\mathrm{n}=5)$, and Enterococcus casseliflavus ( $\mathrm{n}=3$ ). Single isolates of Enterococcus gilvus, Enterococcus devriesei, Enterococcus malodoratus, and Enterococcus villorum were also collected.

The AMR phenotypes of confirmed E. coli (Table 2) and Enterococcus spp. isolates (Table 3) were associated with the antibiotic selective pressure used for culture-based isolation. Escherichia coli isolates were resistant to as many as 10 antibiotics tested (Table 4), belonging to as many as 8 different antibiotic classes (Figure 1). Seventy-eight percent of isolates were resistant to 2 or fewer antibiotic classes (Figure 1). Resistance to multiple antibiotic classes, typically to certain $\beta$-lactams, quinolones, sulfonamides, phenicols, or tetracyclines, was primarily observed when isolates were selected using CIP or FOX supplementation of the medium used in isolation. Escherichia coli isolated on MAC and MAC-ERY were successfully identified from $36(69 \%)$ and $45(87 \%)$ farms, respectively. Few $(<20 \%$ of isolates) of the MAC and MAC-ERY isolates were resistant to any one of the antibiotics tested, and both of these groups had similar antimicrobial susceptibility profiles. Phenotypic similarities between MAC and MAC-ERY were not unexpected given that ERY and other macrolides are generally not selective against members of the Enterobacteriaceae (Phuc Nguyen et al., 2009). Escherichia coli isolated from MAC-FOX were successfully obtained from $29(56 \%)$ of the farms sampled, and of these isolates, $\geq 20 \%$ were resistant to amoxicillin-clavulanate (20\%), cefazolin (CFZ; 36\%), FOX (24\%), and ampicillin (AMP; 36\%). Escherichia coli isolates selected using MAC-CIP agar were collected from 18 (35\%) of the farms sampled, and $\geq 20 \%$ of these isolates were resistant to amoxicillin-clavulanate (30\%), CFZ (78\%), CIP (91\%), sulfamethoxazole/trimethoprim (SXT; 74\%), AMP (87\%), piperacillin (83\%), chloramphenicol (52\%), nalidixic acid (91\%), and TET $(87 \%)$. Little to no resistance $(<10 \%$ of isolates) was observed in any of the $E$. coli isolates to the aminoglycosides gentamicin and tobramycin, the carbapenem 

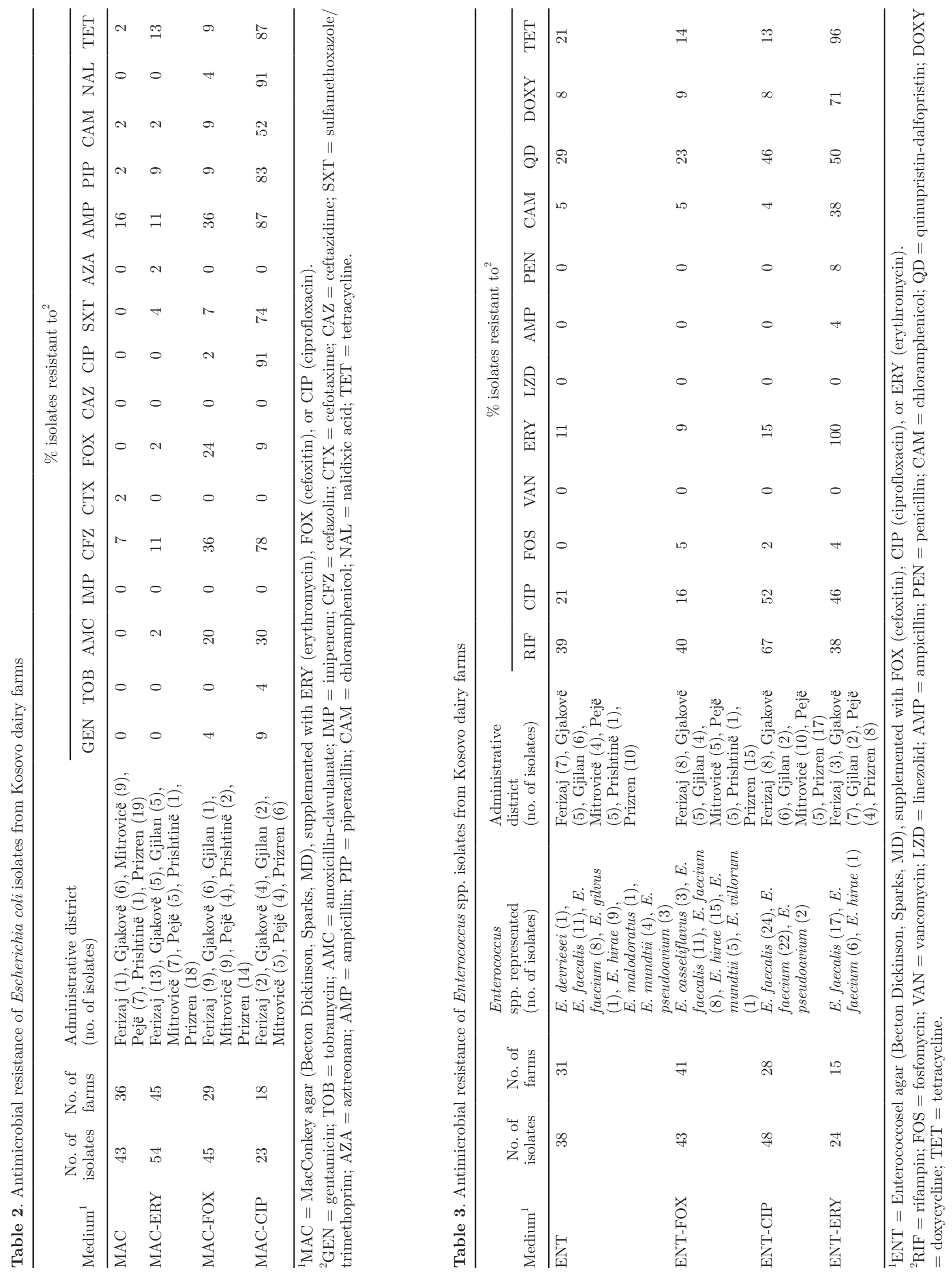
Table 4. Antibiograms of antimicrobial-resistant Escherichia coli isolates from Kosovo dairies

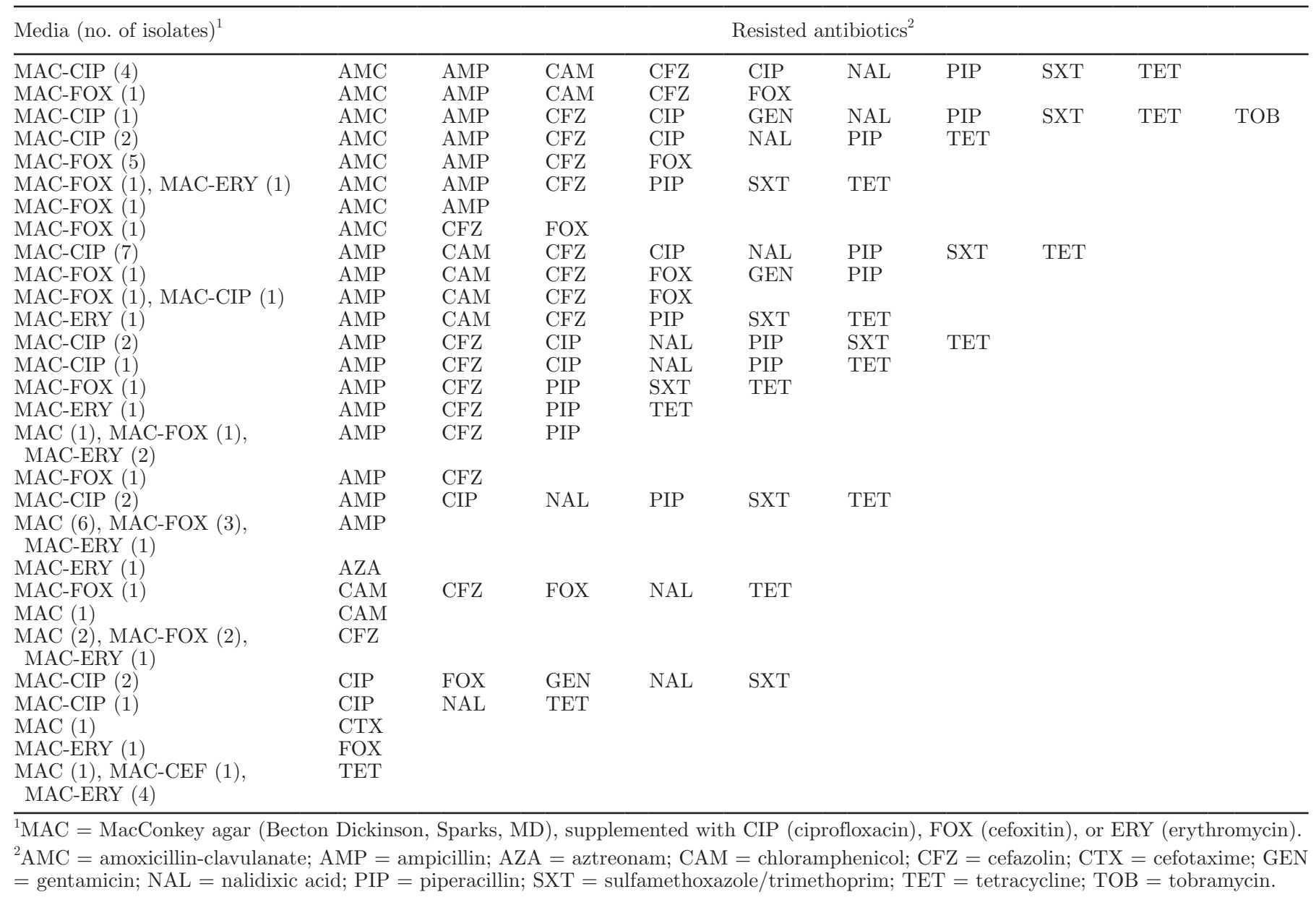

imipenem, the third-generation cephalosporins cefotaxime and ceftazidime, and the monobactam aztreonam.

Interestingly, of the $22 \mathrm{E}$. coli dairy isolates obtained in this study that were resistant to SXT, 20 were also resistant to AMP and TET. Resistance to AMP, SXT, and TET is recognized as a core component of multidrug resistance patterns in E. coli isolates from multiple European countries, potentially reflecting both the past use of these antimicrobials and the frequent association of the genes conferring resistance to these antimicrobials on the same mobile elements (EFSA and ECDC, 2017).

Enterococcus spp. isolates were resistant to as many as 8 antibiotics tested (Table 5), belonging to as many as 6 different antibiotic classes (Figure 1). Of these isolates, $77 \%$ were resistant to 2 or fewer antibiotic classes (Figure 1). Resistance to multiple antibiotic classes, typically to macrolides, tetracyclines, or rifamycins, was primarily observed when isolates were selected for using ERY in the agar medium. Enterococcus spp. isolated on ENT and ENT-FOX were successfully ob- tained from $31(60 \%)$ and $41(79 \%)$ of farms sampled, respectively. Among these isolates, $\geq 39 \%$ were resistant to rifampin (RIF) and $\geq 23 \%$ were resistant to quinupristin-dalfopristin (QD). Given that enterococci are intrinsically resistant to FOX (Moellering et al., 1974), it was anticipated that the antimicrobial susceptibilities in FOX-selected Enterococcus spp. isolates would be similar to those from media without antibiotic supplementation. In general this was observed, although a slightly greater proportion of isolates derived from ENT, compared with isolates from ENT-FOX, were resistant to CIP (21 vs. $16 \%$ of isolates) and TET (21 vs. $14 \%$ of isolates). When ENT-CIP was used for selection, Enterococcus spp. isolates were successfully obtained on $28(54 \%)$ of the farms tested, and $\geq 20 \%$ of these isolates were resistant to RIF (67\%), CIP (52\%), and QD (46\%). Enterococcus spp. isolated using ENT-ERY were found on 15 (29\%) of the farms tested, and $\geq 20$ of these isolates were resistant to RIF (38\%), CIP (46\%), ERY (100\%), chloramphenicol (38\%), QD (50\%), doxycycline $(71 \%)$, and TET (96\%). Little to 
-E. coli $(\mathrm{n}=165) \quad$ Enterococcus spp. $(\mathrm{n}=153)$

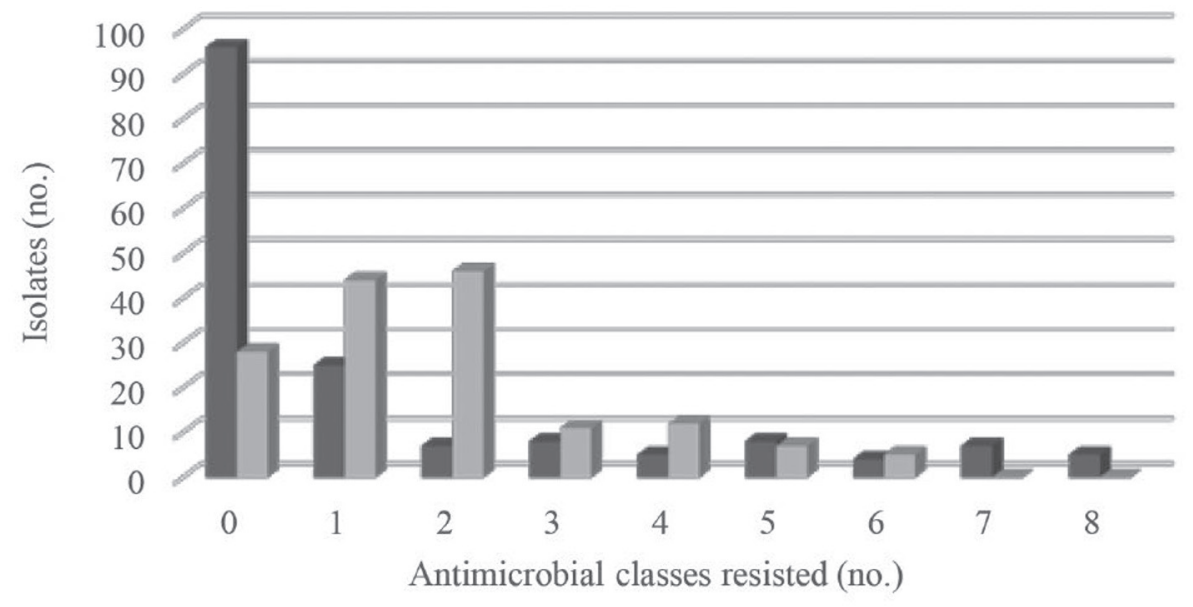

B $\square$ MAC $(n=43) \varpi \operatorname{MAC-FOX~}(\mathrm{n}=45) \varpi \operatorname{MAC}-\mathrm{CIP}(\mathrm{n}=23) \varpi \operatorname{MAC}-E R Y(\mathrm{n}=54)$

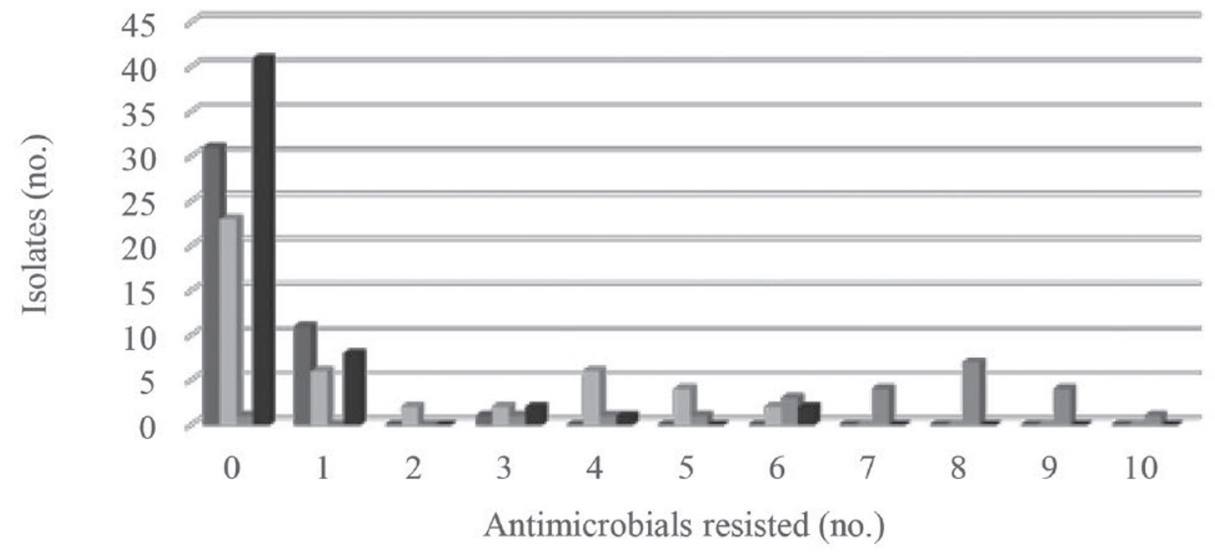

$\mathrm{C} \backsim$ ENT $(\mathrm{n}=38) \llbracket$ ENT-FOX $(\mathrm{n}=43) \square$ ENT-CIP $(\mathrm{n}=48) \square$ ENT-ERY $(\mathrm{n}=24)$

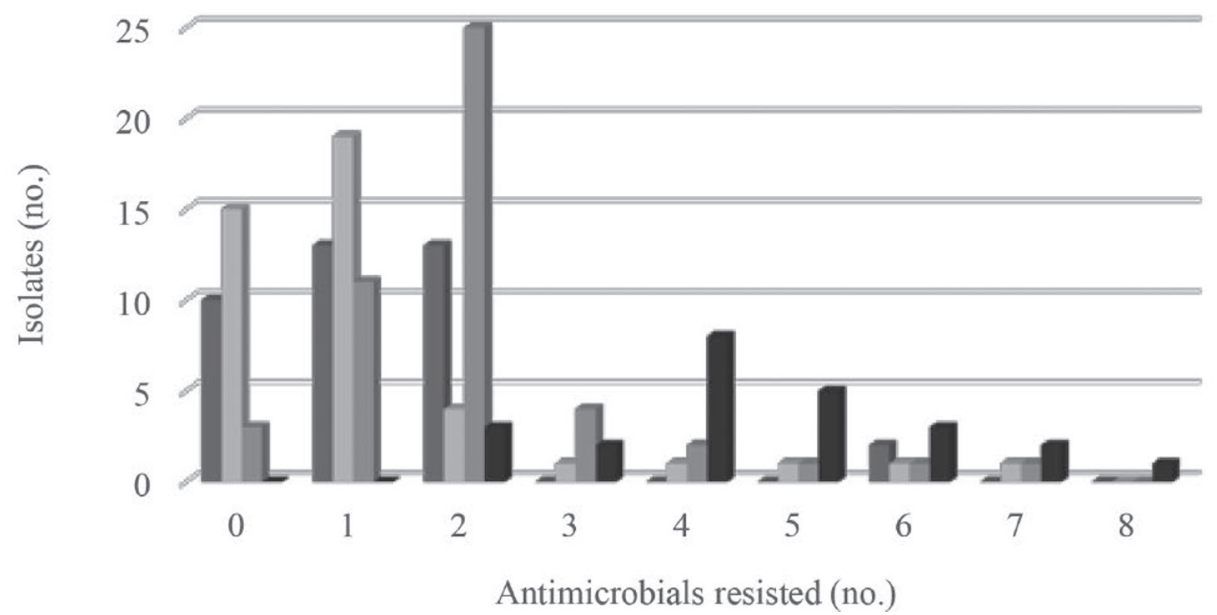

Figure 1. (A) The number of antibiotic classes resisted by Escherichia coli and Enterococcus spp. isolates. The number of antibiotics resisted by (B) E. coli isolates (a total of 16 antibiotics tested) and (C) Enterococcus spp. isolates (a total of 12 antibiotics tested) from media with and without supplemented antibiotics. MAC = MacConkey agar (Becton Dickinson, Sparks, MD), ENT = Enterococcosel agar (Becton Dickinson), $\mathrm{FOX}=$ cefoxitin, $\mathrm{CIP}=$ ciprofloxacin, $\mathrm{ERY}=$ erythromycin. 
Table 5. Antibiograms of antimicrobial-resistant Enterococcus spp. isolates from Kosovo dairies

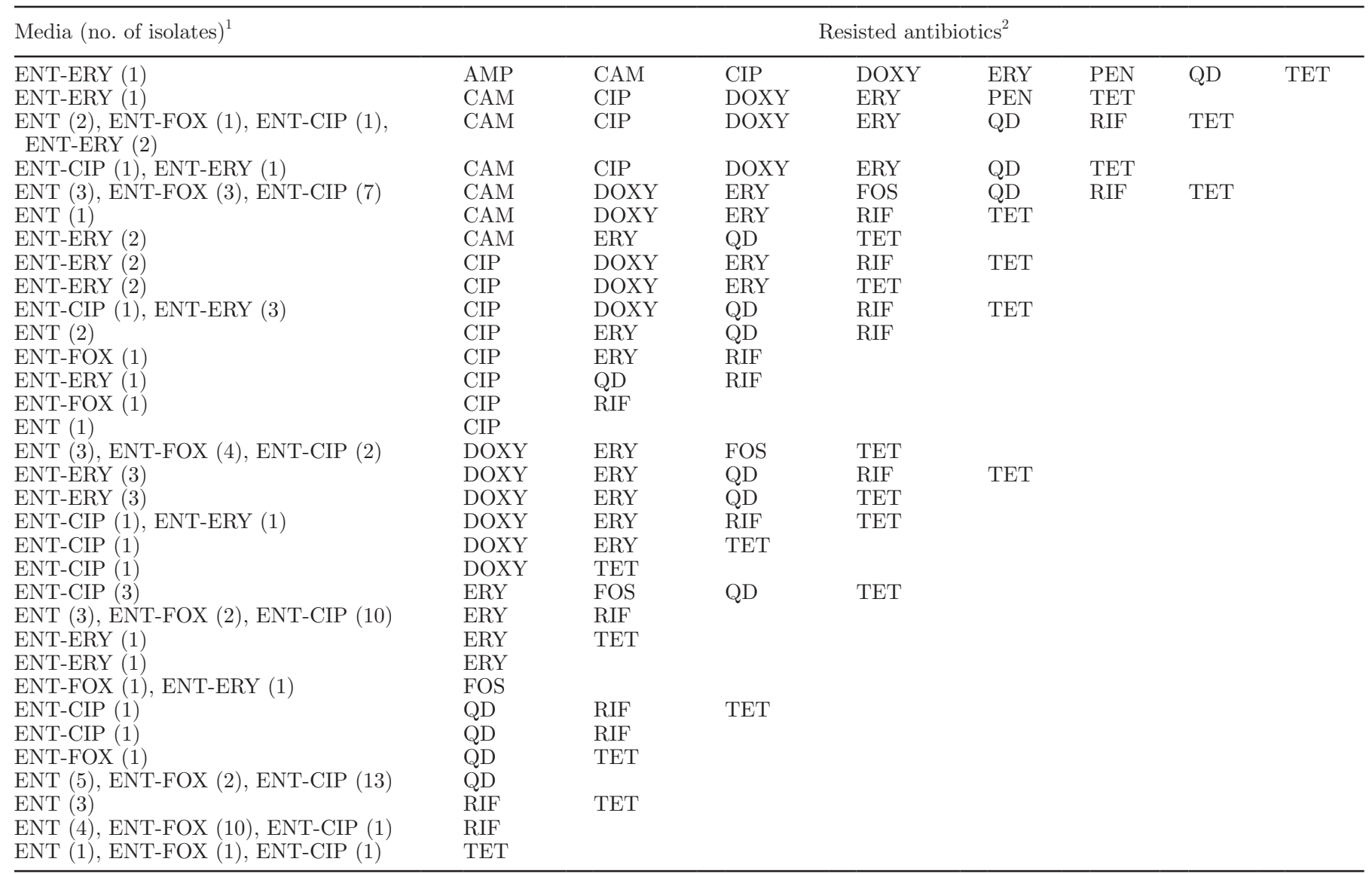

${ }^{1} \mathrm{ENT}=$ Enterococcosel agar (Becton Dickinson, Sparks, MD), supplemented with CIP (ciprofloxacin), FOX (cefoxitin), or ERY (erythromycin) ${ }^{2} \mathrm{AMP}=$ ampicillin; $\mathrm{CAM}=$ chloramphenicol; DOXY $=$ doxycycline; FOS = fosfomycin; PEN = penicillin; QD = quinupristin-dalfopristin; RIF $=$ rifampin; $\mathrm{TET}=$ tetracycline.

no resistance ( $<10 \%$ of isolates) was observed in any of the Enterococcus spp. isolates to FOS, the glycopeptide vancomycin, the oxazolidinone linezolid, and the $\beta$-lactams AMP and penicillin.

Of the epidemiological factors that could possibly be related to the priority AMR phenotypes examined here, only herd size was significant. The odds of observing CIP-resistant E. coli increased by $9 \%$ for every 10 additional cows in a herd (odds ratio of 1.094, 95\% CI 1.007 to 1.188). For Enterococcus spp., the odds of observing ERY-resistant isolates increased by $14 \%$ for every 10 additional cows in a herd (odds ratio of $1.142,95 \%$ CI 1.039 to 1.254 ).

Improved monitoring is needed to better understand the risk associated with the potential transmission of AMR bacteria from food animals to humans. In developing countries, little to no data on AMR or the spread and distribution of zoonotic agents are available. This study provides the first description of AMR in E. coli and Enterococcus spp. isolated from dairy farms across a wide geographical area of Kosovo. These isolates from dairy animals were shown to contain priority AMR phenotypes including cephem, quinolone, and macrolide resistance. Additionally, this study contains the most comprehensive description of AMR phenotypes for any group of bacterial isolates of animal origin collected from Kosovo. Our study provides data on AMR in Kosovo's dairy production, which can be used in future risk assessment studies of AMR in the country's primary production and to aid in informing policy decisions for agricultural antimicrobial use.

\section{ACKNOWLEDGMENTS}

Funding for this project was provided by the University of Wyoming College of Agriculture and Natural Resources Global Perspectives Grant Program. S. F. Aljasir was supported by a graduate scholarship from Qassim University, Saudi Arabia. We thank the dairy farmers of Kosovo for their participation in this study. 


\section{REFERENCES}

ASK (Agjencia E Statisikave Të Kosovës). 2015. Agriculture Census in the Republic of Kosovo 2014-Final Results. Accessed Jan. 28, 2018. http://ask.rks-gov.net/media/1375/final-results.pdf.

Bizzini, A., C. Durussel, J. Bille, G. Greub, and G. Prod'hom. 2010. Performance of matrix-assisted laser desorption ionization-time of flight mass spectrometry for identification of bacterial strains routinely isolated in a clinical microbiology laboratory. J. Clin. Microbiol. 48:1549-1554.

CLSI (Clinical and Laboratory Standards Institute). 2015. M02-A12: Performance standards for antimicrobial disk susceptibility tests; Approved Standard-Twelfth Edition. CLSI, Wayne, PA.

EFSA and ECDC (European Food Safety Authority and European Centre for Disease Prevention and Control). 2017. The European Union summary report on antimicrobial resistance in zoonotic and indicator bacteria from humans, animals and food in 2015. EFSA J. 15:4694. https://doi.org/10.2903/j.efsa.2017.4694.

Founou, L. L., R. C. Founou, and S. Y. Essack. 2016. Antibiotic resistance in the food chain: A developing country-perspective. Front. Microbiol. 7:1881.

Frye, J. G., and C. R. Jackson. 2013. Genetic mechanisms of antimicrobial resistance identified in Salmonella enterica, Escherichia coli, and Enteroccocus spp. isolated from U.S. food animals. Front. Microbiol. 4:135.

Gallina, G., A. Rama, L. Lucatello, C. Benetti, D. Bajraktari, K. Uka, and C. Montesissa. 2013. Aflatoxin $\mathrm{M}_{1}$ contamination and antibacterial residues in milk in Kosovo. Trends in Veterinary Sciences: Current Aspects in Veterinary Morphophysiology, Biochemistry, Animal Production, Food Hygiene and Clinical Sciences. C. Boiti, A. Ferlazzo, A. Gaiti, and A. Pugliese, ed. Springer, Heidelberg, Germany.

Hamidi, A., and D. Sylejmani. 2016. Antimicrobial susceptibility of staphylococci isolated from clinical mastitis in dairy cows in Kosovo. Bulg. J. Agric. Sci. 22:836-839.

Harwood, V. J., J. Whitlock, and V. Withington. 2000. Classification of antibiotic resistance patterns of indicator bacteria by discriminant analysis: Use in predicting the source of fecal contamination in subtropical waters. Appl. Environ. Microbiol. 66:3698-3704.

Lillehaug, A., B. Bergsjo, J. Schau, T. Bruheim, T. Vikoren, and K. Handeland. 2005. Campylobacter spp., Salmonella spp., verocytotoxic Escherichia coli, and antibiotic resistance in indicator organisms in wild cervids. Acta Vet. Scand. 46:23-32.

Mehmeti, I., B. Behluli, M. Mestani, A. Ademi, I. F. Nes, and D. B. Diep. 2016. Antimicrobial resistance levels amongst staphylococci isolated from clinical cases of bovine mastitis in Kosovo. J. Infect. Dev. Ctries. 10:1081-1087.

Mehmeti, I., S. Muji, D. B. Diep, and I. F. Nes. 2015. High frequency of the potential pathogen Lactococcus garvieae in raw milk from Kosovo. Food Control 53:189-194.

Moellering, R. C. Jr., M. Dray, and L. J. Kunz. 1974. Susceptibility of clinical isolates of bacteria to cefoxitin and cephalothin. Antimicrob. Agents Chemother. 6:320-323.

Phuc Nguyen, M. C., P. L. Woerther, M. Bouvet, A. Andremont, R Leclercq, and A. Canu. 2009. Escherichia coli as reservoir for macrolide resistance genes. Emerg. Infect. Dis. 15:1648-1650.

Sulejmani, Z., A. Shehi, Z. Hajrulai, and E. Mata. 2012. Abuse of pharmaceutical drugs-Antibiotics in dairy cattle in Kosovo and detection of their residues in milk. Pharmacologyonline 1:30-38.

US AID. 2007: Kosovo dairy value chain case study; Guided case studies in value chain development for conflict-affected environments; microREPORT \#95. Accessed Jan. 28, 2018. http://pdf.usaid .gov/pdf_docs/Pnadn387.pdf.

US CDC. 2013. Antibiotic resistance threats in the United States, 2013. Accessed Aug. 2, 2017. https://www.cdc.gov/drugresistance/pdf/ ar-threats-2013-508.pdf.

US FDA. 2016. 2015 summary report of antimicrobial sold or distributed for use in food-producing animals. Accessed August 2, 2017. https://www.fda.gov/downloads/ForIndustry/UserFees/ AnimalDrugUserFeeActADUFA/UCM534243.pdf.

US FDA-NARMS. 2014. 2012 Retail meat report. Accessed Aug. 2, 2017. https://www.fda.gov/downloads/ AnimalVeterinary/SafetyHealth/AntimicrobialResistance/ NationalAntimicrobialResistanceMonitoringSystem/UCM442212 .pdf.

Van Boeckel, T. P., C. Brower, M. Gilbert, B. T. Grenfell, S. A. Levin, T. P. Robinson, A. Teillant, and R. Laxminarayan. 2015. Global trends in antimicrobial use in food animals. Proc. Natl. Acad. Sci. USA 112:5649-5654.

Versporten, A., G. Bolokhovets, L. Ghazaryan, V. Abilova, G. Pyshnik, T. Spasojevic, I. Korinteli, L. Raka, B. Kambaralieva, L. Cizmovic, A. Carp, V. Radonjic, N. Maqsudova, H. D. Celik, M. Payerl-Pal, H. B. Pedersen, N. Sautenkova, and H. Goossens.WHO/ Europe-ESAC Project Group. 2014. Antibiotic use in eastern Europe: A cross-national database study in coordination with the WHO Regional Office for Europe. Lancet Infect. Dis. 14:381-387.

WHO. 2014. Antimicrobial resistance: Global report on surveillance, 2014. Accessed Aug. 2, 2017. http://apps.who.int/iris/bitstream/ 10665/112642/1/9789241564748_eng.pdf?ua=1. 